\title{
A note on global units and local units of function fields
}

\author{
by
}

Su Hu and YAN Li (Beijing)

1. Introduction. Let $K$ be any Galois extension of $\mathbb{Q}$, and $U_{K}$ be the unit group of $K$. For any place $v$ of $K$, let $U_{v}$ be the group of local units of $K_{v}$. Recently, the second author and Xianke Zhang [6] considered the problem of whether there exists an odd prime $p$ such that the map

$$
U_{K} / U_{K}^{2} \rightarrow \prod_{v \mid p} U_{v} / U_{v}^{2}
$$

is injective. In fact, they proved that the existence of such primes is equivalent to $\operatorname{Hom}\left(U_{K} / U_{K}^{2},\{ \pm 1\}\right)$ is a cyclic $\mathbb{F}_{2}[\operatorname{Gal}(K / \mathbb{Q})]$-module. Moreover, they also proved that if the class number $h_{\mathbb{Q}\left(\zeta_{p^{r}}\right)^{+}}$is odd, then such primes exist for $\mathbb{Q}\left(\zeta_{p^{r}}\right)^{+}$and $\mathbb{Q}\left(\zeta_{p^{r}}\right)$, where $p$ is an odd prime and $\mathbb{Q}\left(\zeta_{p^{r}}\right)^{+}$is the maximal real subfield of $\mathbb{Q}\left(\zeta_{p^{r}}\right)$.

Let $K$ be a geometric Galois extension of the rational function field $k=\mathbb{F}_{q}(t)$. Let $O_{K}$ be the integral closure of $\mathbb{F}_{q}[t]$ in $K$. Let $U_{K}$ be the group of units of $O_{K}$ and $U_{v}$ be the group of local units of $K_{v}$. In this note, we will generalize the second author and Zhang's methods to consider the question whether there exists a finite place $P$ of $\mathbb{F}_{q}(t)$ such that the map

$$
U_{K} / U_{K}^{d} \rightarrow \prod_{v \mid P} U_{v} / U_{v}^{d}
$$

is injective, where $d>1$ is a factor of $q-1$. Let $\mu_{d}$ be the group of $d$ th roots of unity. We will prove there exist such places $P$ if and only if $\operatorname{Hom}\left(U_{K} / U_{K}^{d}, \mu_{d}\right)$ is a cyclic $\mathbb{Z} / d \mathbb{Z}[\operatorname{Gal}(K / k)]$-module. When $K$ is a quadratic function field, we will prove in Section 4 that there exist such places if and only if either $K$ is imaginary, or $K$ is real and $d$ is odd, or $K$ is real, $d$ is even and there exists a fundamental unit $\epsilon_{0}$ of $O_{K}$ such that $N\left(\epsilon_{0}\right)$ is a generator of $\mathbb{F}_{q}^{*}$. Let $A$ be a monic irreducible polynomial. Suppose that $K=k\left(\Lambda_{A}\right)$ is the $A$ th cyclotomic function field and $K^{+}$is the maximal real subfield of $K$. In 
Section 5, we will prove that such places exist for $K$ and $K^{+}$if the class number of $O_{K^{+}}$is relatively prime to $d$. It should be noted that the proof heavily relies on Galovich and Rosen's work on Sinnott's circular units in cyclotomic function fields [3].

2. Preliminaries. For each $d \mid q-1$, define $L=K\left(\sqrt[d]{U_{K}}\right)$. Since $\mathbb{F}_{q}$ contains $d$ th roots of unity, $L$ is an abelian extension of $K$ of exponent $d$. Set $\operatorname{Gal}(L / K)=H$ and $\operatorname{Gal}\left(K / \mathbb{F}_{q}(t)\right)=G$. Define an action of $G$ on $H$ by $g h=\tilde{g} h \tilde{g}^{-1}$, where $g \in G, h \in H$ and $\tilde{g}$ is a lift of $g$ in $\operatorname{Gal}\left(L / \mathbb{F}_{q}(t)\right)$. By Kummer theory (e.g. Theorem 8.1 of [5]), there is a non-degenerate $G$-equivariant bilinear pairing

$$
H \times U_{K} / U_{K}^{d} \rightarrow \mu_{d}, \quad(h, \bar{u})=\frac{h(\sqrt[d]{u})}{\sqrt[d]{u}} .
$$

Therefore we have $H \cong \operatorname{Hom}\left(U_{K} / U_{K}^{d}, \mu_{d}\right)$ as $G$-modules. The action of $G$ on $\operatorname{Hom}\left(U_{K} / U_{K}^{d}, \mu_{d}\right)$ is defined by

$$
g f(\bar{u})=f\left(g^{-1} \bar{u}\right)
$$

for $g \in \operatorname{Gal}\left(K / \mathbb{F}_{q}(t)\right), f \in \operatorname{Hom}\left(U_{K} / U_{K}^{d}, \mu_{d}\right), \bar{u} \in U_{K} / U_{K}^{d}$.

Assume the infinite place $(1 / t)$ of $\mathbb{F}_{q}(t)$ splits into $r$ places of $K$. By Dirichlet's unit theorem, the rank of $U_{K} / U_{K}^{d}$ as $\mathbb{Z} / d \mathbb{Z}$-module is equal to $r$. Let $\left\{u_{1}, \ldots, u_{r}\right\} \subset U_{K}$ be representatives such that $\bar{u}_{1}, \ldots, \bar{u}_{r}$ form a $\mathbb{Z} / d \mathbb{Z}$ basis of $U_{K} / U_{K}^{d}$. Then it is easy to show that

$$
H \simeq \operatorname{Gal}\left(K\left(\sqrt[d]{u_{1}}\right) / K\right) \times \cdots \times \operatorname{Gal}\left(K\left(\sqrt[d]{u_{r}}\right) / K\right) .
$$

The isomorphism is given by restriction to the subfields.

The following is Chebotarev's density theorem for global function fields (Theorem 9.13A of [7]).

TheOREM 2.1 (Chebotarev). Let $L / K$ be a Galois extension of global function fields and $\operatorname{Gal}(L / K)=H$. Let $C \subset H$ be a conjugacy class and $S_{K}^{\prime}$ be the set of primes of $K$ which are unramified in $L$. Then

$$
\delta\left(\left\{\mathfrak{p} \in S_{K}^{\prime} \mid(\mathfrak{p}, L / K)=C\right\}\right)=\# C / \# H,
$$

where $\delta$ means Dirichlet density. In particular, every conjugacy class $C$ is of the form $(\mathfrak{p}, L / K)$ for infinitely many places $\mathfrak{p}$ of $K$.

Lemma 2.2. Let $u \in U_{K}$ and $\mathfrak{p}$ be a place of $K$ which is unramified in $L$. Then $u \in U_{\mathfrak{p}}^{d}$ if and only if $(\mathfrak{p}, L / K)$ fixes $K(\sqrt[d]{u})$, where $L=K\left(\sqrt[d]{U_{K}}\right)$ (see the beginning of this section).

Proof. $u \in U_{\mathfrak{p}}^{d}$ is equivalent to $\mathfrak{p}$ splitting completely in $K(\sqrt[d]{u})$. Since $\mathfrak{p}$ is unramified in $K(\sqrt[d]{u})$, this is equivalent to $(\mathfrak{p}, K(\sqrt[d]{u}) / K)=\operatorname{Id}$. As the Artin symbol satisfies $\left.(\mathfrak{p}, L / K)\right|_{K(\sqrt[d]{u})}=(\mathfrak{p}, K(\sqrt[d]{u}) / K)$, the result follows. 


\section{Proof of the main result}

Proposition 3.1. The natural map $U_{K} / U_{K}^{d} \rightarrow \prod_{v} U_{v} / U_{v}^{d}$ is injective, where $v$ runs over all finite places of $K$.

Proof. Let $u$ belong to the kernel of the map. Then $u \in U_{v}^{d}$ for all $v$. By Lemma 2.2, $(v, K(\sqrt[d]{u}) / K)=$ Id for all finite places $v$. Consequently, $\delta\left(\left\{\mathfrak{p} \in S_{K}^{\prime} \mid(\mathfrak{p}, L / K)=\operatorname{Id}\right\}\right)=1$. By Chebotarev's density theorem, the extension $K(\sqrt[d]{u}) / K$ is trivial. Thus $u \in U_{K}^{d}$.

Proposition 3.2. There exist places $\mathfrak{p}_{1}, \ldots, \mathfrak{p}_{r}$ of $K$ such that the natural map

is injective.

$$
U_{K} / U_{K}^{d} \rightarrow \prod_{1 \leq i \leq r} U_{\mathfrak{p}_{i}} / U_{\mathfrak{p}_{i}}^{d}
$$

Proof. Let $\sigma_{1}, \ldots, \sigma_{r} \in H$ be such that the restriction of $\sigma_{i}$ to $K\left(\sqrt[d]{u_{j}}\right)$ is trivial when $j \neq i$ and is a generator of $\operatorname{Gal}\left(K\left(\sqrt[d]{u_{j}}\right) / K\right)$ for $j=i$. By Chebotarev's density theorem, there exist finite places $\mathfrak{p}_{1}, \ldots, \mathfrak{p}_{r}$ of $K$ such that $\left(\mathfrak{p}_{i}, L / K\right)=\sigma_{i}$. If $u$ belongs to the kernel, then $u \in U_{K_{\mathfrak{p}_{i}}}^{d}$. By Lemma 2.2, $\sigma_{i}$ fixes $K(\sqrt[d]{u})$. By construction, $\sigma_{1}, \ldots, \sigma_{r}$ generate $H$, so $K(\sqrt[d]{u})=K$ by Galois theory. Thus $u \in U_{K}^{d}$.

Proposition 3.3. Let $P$ be a finite place of $\mathbb{F}_{q}(t)$. Then the natural map

$$
U_{K} / U_{K}^{d} \rightarrow \prod_{v \mid P} U_{v} / U_{v}^{d}
$$

is injective if and only if for some place $v \mid P$ (hence for all $v \mid P),(v, L / K)$ is a $\mathbb{Z} / d \mathbb{Z}[G]$ generator of $H$.

Proof. Let $u$ be any unit of $K$. By Lemma 2.2,

$$
u \in U_{v}^{d}, \forall v \mid P \Leftrightarrow(v, L / K) \text { fixes } K(\sqrt[d]{u}), \forall v \mid P .
$$

It is obvious that

$$
u \in U_{K}^{d} \Leftrightarrow K(\sqrt[d]{u})=K .
$$

Thus, $U_{K} / U_{K}^{d} \rightarrow \prod_{v \mid P} U_{v} / U_{v}^{d}$ being injective is equivalent to

$$
\forall u \in U_{K},(v, L / K) \text { fixes } K(\sqrt[d]{u}), \forall v \mid P \Rightarrow K(\sqrt[d]{u})=K .
$$

By Galois theory, this is equivalent to the subgroup generated by $(v, L / K)$ for all $v \mid P$ being equal to $H$. Recall the definition of the action of $G$ on $H$ in Section 2: $(g v, L / K)=\tilde{g}(v, L / K) \tilde{g}^{-1}=g(v, L / K)$. This is also equivalent to $(v, L / K)$ being a $\mathbb{Z} / d \mathbb{Z}[G]$ generator of $H$ for any $v \mid P$.

Theorem 3.4. There exists a finite place $P$ of $\mathbb{F}_{q}(t)$ such that the map

$$
U_{K} / U_{K}^{d} \rightarrow \prod_{v \mid P} U_{v} / U_{v}^{d}
$$

is injective if and only if $\operatorname{Hom}\left(U_{K} / U_{K}^{d}, \mu_{d}\right)$ is a cyclic $\mathbb{Z} / d \mathbb{Z}[G]$-module. 
Proof. Since $H$ is isomorphic to $\operatorname{Hom}\left(U_{K} / U_{K}^{d}, \mu_{d}\right)$ as $\mathbb{Z} / d \mathbb{Z}[G]$-modules, the "only if" part follows easily from Proposition 3.3. Conversely, if $H$ is a cyclic module, let $\sigma \in H$ be a $\mathbb{Z} / d \mathbb{Z}[G]$ generator of $H$. By Chebotarev's density theorem, there exists a finite place $\mathfrak{p}$ such that $(\mathfrak{p}, L / K)=\sigma$. Also by Proposition 3.3, we conclude that $U_{K} / U_{K}^{d} \rightarrow \prod_{g \in G} U_{g \mathfrak{p}} / U_{g \mathfrak{p}}^{d}$ is injective.

The following definition can be found on page 371 of [8].

Definition 3.5. An extension $K$ of $k=\mathbb{F}_{q}(t)$ is called totally real if the prime at infinity of $k$ (which corresponds to $1 / t$ ) splits completely in $K$.

Lemma 3.6. Let $G$ be a finite group and $V$ be a free $\mathbb{Z} / d \mathbb{Z}$-module of rank $r=\# G$. Assume $G$ acts on $V$ linearly. Then $V$ is a cyclic $\mathbb{Z} / d \mathbb{Z}[G]$-module if and only if $V^{*}=\operatorname{Hom}(V, \mathbb{Z} / d \mathbb{Z})$ is a cyclic $\mathbb{Z} / d \mathbb{Z}[G]$-module.

TheOrem 3.7. If $K$ is a totally real geometric Galois extension of $\mathbb{F}_{q}(t)$, there exists a finite place $P$ of $\mathbb{F}_{q}(t)$ such that the natural map

$$
U_{K} / U_{K}^{d} \rightarrow \prod_{v \mid P} U_{v} / U_{v}^{d}
$$

is injective if and only if $U_{K} / U_{K}^{d}$ is a cyclic $\mathbb{Z} / d \mathbb{Z}[G]$-module.

Proof. Suppose that $\left[K: \mathbb{F}_{q}(t)\right]=n$. By Definition 3.5, $K$ has $n$ infinite places. By Dirichlet's unit theorem, $U_{K} / U_{K}^{d}$ is a free $\mathbb{Z} / d \mathbb{Z}$-module of rank $n$. By Theorem 3.4, the injectivity in question is equivalent to $\operatorname{Hom}\left(U_{K} / U_{K}^{d}, \mu_{d}\right)$ being a cyclic $\mathbb{Z} / d \mathbb{Z}[G]$-module. Applying Lemma 3.6 to $V=U_{K} / U_{K}^{d}$, we get the desired result.

A unit $u$ is called a Minkowski unit if its Galois conjugates generate a subgroup of finite index in the whole unit group. We know that such units always exist (see [9, Lemma 5.27], the proof is the same for global function fields).

Corollary 3.8. Let $K / \mathbb{F}_{q}(t)$ be a totally real geometric Galois extension. There exists a finite place $P$ of $\mathbb{F}_{q}(t)$ such that the natural map

$$
U_{K} / U_{K}^{d} \rightarrow \prod_{v \mid P} U_{v} / U_{v}^{d}
$$

is injective if and only if there exists a Minkowski unit $\epsilon$ such that the index of $\mathbb{Z}[G] \epsilon$ in $U_{K}$ is relatively prime to $d$.

Proof. By Theorem 3.4, the existence of such $P$ is equivalent to $U_{K} / U_{K}^{d}$ being a cyclic $\mathbb{Z} / d \mathbb{Z}[G]$-module. This means that there exists a unit $\epsilon$ such that $U_{K}=U_{K}^{d}(\mathbb{Z}[G] \epsilon)$. Let $E=\mathbb{Z}[G] \epsilon$. We get

$$
U_{K}=E U_{K}^{d} \Leftrightarrow U_{K} / E=\left(U_{K} / E\right)^{d} \Leftrightarrow\left(\# U_{K} / E, d\right)=1 .
$$

This completes the proof of the corollary. 
4. The case of quadratic function fields. In this section, we assume $K$ is a quadratic extension of $k=\mathbb{F}_{q}(t)$ and $2 \nmid q$. We will use the theory developed in Section 3 to investigate the situation of quadratic function fields. Such fields can be written as $k(\sqrt{D})$, where $D$ is a square free polynomial of $\mathbb{F}_{q}[t]$. They were systematically studied by E. Artin [1].

Fix a generator $g$ of $\mathbb{F}_{q}^{*}$. Then we can assume that the leading coefficient of $D$ is 1 or $g$. The infinite place $(1 / t)$ is splitting, inertial, or ramified in $K$ when, respectively: the degree of $D$ is even and $\operatorname{sgn}(D)=1$; the degree of $D$ is even and $\operatorname{sgn}(D)=g$; or the degree of $D$ is odd. Then the field $K$ is called real, inertial imaginary, or ramified imaginary respectively, according to E. Artin [1]. When $K$ is real, we let $\epsilon_{0}$ be the fundamental unit of $K$. Any fundamental unit is determined only up to multiplication by a constant, thus its norm is either a square or $g$ times a square. So multiplying $\epsilon_{0}$ by an appropriate constant we can assume $N\left(\epsilon_{0}\right)$ is 1 or $g$.

Now we state the main theorem of this section.

TheOREM 4.1. Let the notations be as above. There exists a finite place $P$ of $\mathbb{F}_{q}(t)$ such that

$$
U_{K} / U_{K}^{d} \rightarrow \prod_{v \mid P} U_{v} / U_{v}^{d}
$$

is injective if and only if either $K$ is imaginary, or $K$ is real and $d$ is odd, or $K$ is real, $d$ is even and $N\left(\epsilon_{0}\right)=g$.

Proof. If $K$ is imaginary, then $U_{K}=\mathbb{F}_{q}^{*}$ and $U_{K} / U_{K}^{d}=\mathbb{F}_{q}^{*} / \mathbb{F}_{q}^{* d}$ is a cyclic group. Thus $\operatorname{Hom}\left(U_{K} / U_{K}^{d}, \mathbb{Z} / d \mathbb{Z}\right)$ is a cyclic $\mathbb{Z} / d \mathbb{Z}[G]$-module. By Theorem 3.4, there exists a finite place $P$ of $\mathbb{F}_{q}(t)$ such that

$$
U_{K} / U_{K}^{d} \rightarrow \prod_{v \mid P} U_{v} / U_{v}^{d}
$$

is injective.

If $K$ is real, then $U_{K}=\left\langle\epsilon_{0}\right\rangle \times \mathbb{F}_{q}^{*}$. By Corollary 3.8, the existence of such places is equivalent to the existence of a Minkowski unit $\epsilon$ such that $\left(\# U_{K} / \mathbb{Z}[G] \epsilon, d\right)=1$. If $N\left(\epsilon_{0}\right)=g$, we can take $\epsilon=\epsilon_{0}$, and then $U_{K}=\mathbb{Z}[G] \epsilon$. If $N\left(\epsilon_{0}\right)=1$ and $d$ is odd, we can take $\epsilon=g \epsilon_{0}$, and then

$$
\mathbb{Z}[G] \epsilon=\mathbb{Z} \epsilon \oplus\langle N(\epsilon)\rangle=\mathbb{Z} \epsilon \oplus\left\langle g^{2}\right\rangle .
$$

Thus $\# U_{K} / \mathbb{Z}[G] \epsilon=2$ is prime to $d$. If $N\left(\epsilon_{0}\right)=1$ and $d$ is even, for any Minkowski unit $\epsilon$, write $\epsilon=\epsilon_{0}^{k} g^{l}, k, l \in \mathbb{Z}, k \neq 0$. As above,

$$
\mathbb{Z}[G] \epsilon=\mathbb{Z} \epsilon \oplus\langle N(\epsilon)\rangle=\mathbb{Z} \epsilon \oplus\left\langle g^{2 l}\right\rangle \subset \mathbb{Z} \epsilon \oplus\left\langle g^{2}\right\rangle .
$$

Thus $2 \mid \# U_{K} / \mathbb{Z}[G] \epsilon$, so $2 \mid\left(\# U_{K} / \mathbb{Z}[G] \epsilon, d\right)$. The proof is complete. 
5. The case of cyclotomic function fields. Before stating the main theorem of this section, we must introduce some notation. Write $k=\mathbb{F}_{q}(t)$ and $R=\mathbb{F}_{q}[t]$. Let $k^{\text {ac }}$ be the algebraic closure of $k$. In order to construct the explicit class field theory for $k$, Carlitz [2] introduced an $R$-module structure on $k^{\text {ac }}$, called the Carlitz module (see also [4]). Let $\operatorname{End}\left(k^{\mathrm{ac}}\right)$ be the ring of $\mathbb{F}_{q^{-}}$-algebra endomorphisms of $k^{\mathrm{ac}}$. Let

$$
\rho: R \rightarrow \operatorname{End}\left(k^{\mathrm{ac}}\right), \quad M \mapsto \rho_{M},
$$

be a ring homomorphism defined by

$$
\rho_{a}(\alpha)=a \alpha, \quad \rho_{t}(\alpha)=t \alpha+\alpha^{q},
$$

where $a \in \mathbb{F}_{q}$ and $\alpha \in k^{\text {ac }}$. Let

$$
\Lambda_{M}=\left\{\alpha \in k^{\mathrm{ac}} \mid \rho_{M}(\alpha)=0\right\},
$$

which is called the $M$-torsion module of $k^{\mathrm{ac}}$. If $M$ is monic, $k\left(\Lambda_{M}\right)$ is called the $M$ th cyclotomic function field. Chapter 12 of [7] gives a nice exposition of the theory of cyclotomic function fields. Let $S_{\infty}\left(k\left(\Lambda_{M}\right)\right)$ be the set of infinite places of $k\left(\Lambda_{M}\right)$ and $U_{M}$ be the group of $S_{\infty}\left(k\left(\Lambda_{M}\right)\right)$-units of $k\left(\Lambda_{M}\right)$. For simplicity, let $\mathrm{P}(3)$ denote the following property: there exists a finite place $P$ in $\mathbb{F}_{q}(t)$ such that $U_{K} / U_{K}^{d} \rightarrow \prod_{v \mid P} U_{v} / U_{v}^{d}$ is injective, where $K$ is a geometric Galois extension of $\mathbb{F}_{q}(t)$. Now we can state the main theorem of this section.

TheOREM 5.1. Let $A$ be a monic irreducible polynomial in $\mathbb{F}_{q}[t], K=$ $k\left(\Lambda_{A}\right)$ and $K^{+}$be the maximal real subfield of $K$ (for the definitions, see Theorem 12.14 of [7]). Let $h_{A}$ be the class number of $O_{K}$ and $h_{A}^{+}$be the class number of $O_{K}^{+}$. If $d \mid q-1$ and $\left(h_{A}^{+}, d\right)=1$, then $\mathrm{P}(3)$ holds for $K$ and $K^{+}$.

Before proving the above theorem, we briefly recall Galovich and Rosen's work on Sinnott's cyclotomic units in cyclotomic function fields [3].

Definition 5.2. Let $M$ be a monic polynomial in $\mathbb{F}_{q}[t]$, and $\lambda$ be a primitive $M$-torsion element. Define

$S=\left\{\rho_{B}(\lambda) / \lambda \mid B\right.$ is a monic polynomial, $\left.0<\operatorname{deg} B<\operatorname{deg} M,(B, M)=1\right\}$

(obviously, $S \subset U_{k\left(\Lambda_{M}\right)^{+}}$). The elements in the subgroup generated by $S$ are called the Kummer-Hilbert circular units, denoted by $C_{y}\left(k\left(\Lambda_{M}\right)^{+}\right)$. Let $G$ be the multiplicative subgroup of $k\left(\Lambda_{M}\right)^{*}$ generated by $\mathbb{F}_{q}^{*}$ and $\Lambda_{M}^{*}=\Lambda_{M}-\{0\}$. The elements of $C=G \cap U_{k\left(\Lambda_{M}\right)}$ and $C^{+}=C \cap U_{k\left(\Lambda_{M}\right)^{+}}$are called the Sinnott circular units of $k\left(\Lambda_{M}\right)$ and $k\left(\Lambda_{M}\right)^{+}$, respectively.

Remark 5.3. Since $A$ is irreducible, from [3] we know that

$$
U_{K}=U_{K^{+}}, \quad C=C^{+} \quad \text { and } \quad C^{+}=\mathbb{F}_{q}^{*} C_{y}\left(K^{+}\right) .
$$


In this case, Galovich and Rosen proved (see [3])

Theorem 5.4 (Galovich-Rosen). $\left[U_{K}: C\right]=\left[U_{K^{+}}: C^{+}\right]=h_{A}^{+}$.

Now we can start the proof of Theorem 5.1.

Proof of Theorem 5.1. Let

$$
G=\operatorname{Gal}(K / k) \text { and } G^{+}=\operatorname{Gal}\left(K^{+} / k\right) .
$$

From Remark 5.3, we have $U_{K} / U_{K}^{d}=U_{K^{+}} / U_{K^{+}}^{d}$. Thus $\operatorname{Hom}\left(U_{K} / U_{K}^{d}, \mu_{d}\right)$ is a cyclic $\mathbb{Z} / d \mathbb{Z}[G]$-module if and only if $\operatorname{Hom}\left(U_{K^{+}} / U_{K^{+}}^{d}, \mu_{d}\right)$ is a cyclic $\mathbb{Z} / d \mathbb{Z}\left[G^{+}\right]$-module. By Theorem 3.4, $\mathrm{P}(3)$ holds for $K$ if and only if $\mathrm{P}(3)$ holds for $K^{+}$. From Theorem $5.4, h_{A}^{+}=\left[U_{K^{+}}: C^{+}\right]$, so $\left(\left[U_{K^{+}}: C^{+}\right], d\right)=1$ by assumption. If we can show that $C^{+}$is a cyclic $\mathbb{Z} / d \mathbb{Z}\left[G^{+}\right]$-module, then by Corollary 3.8, we will complete the proof. Suppose $M$ is a generator of $\left(\mathbb{F}_{q}[t] / A \mathbb{F}_{q}[t]\right)^{*}$. Since by Remark $5.3, C^{+}=\mathbb{F}_{q}^{*} C_{y}\left(K^{+}\right), C^{+}$is generated by the set

$$
\tilde{S}=\left\{\rho_{M^{i}}(\lambda) / \lambda \mid 1 \leq i \leq q^{\operatorname{deg} A}-1\right\} .
$$

For each polynomial $W$ relatively prime to $A$ there is a unique element $\sigma_{W} \in G$ such that $\sigma_{W}(\lambda)=\rho_{W}(\lambda)$ where $\lambda$ is a primitive $A$-torsion element (see Theorem 12.8 of [7]). Using the definition of the group ring action, the multiplicity of $\sigma_{N}$, and cancellation in a telescoping product, we have

$$
\begin{aligned}
\frac{\rho_{M^{i+1}}(\lambda)}{\lambda} & =\frac{\sigma_{M^{i+1}}(\lambda)}{\lambda} \\
& =\left(1+\sigma_{M}+\sigma_{M^{2}}+\cdots+\sigma_{M^{i}}\right) \frac{\sigma_{M}(\lambda)}{\lambda} .
\end{aligned}
$$

Thus

$$
C^{+}=\mathbb{Z}[G] \frac{\sigma_{M}(\lambda)}{\lambda}=\mathbb{Z}\left[G^{+}\right] \frac{\sigma_{M}(\lambda)}{\lambda}
$$

is a cyclic module, and the proof is finished.

Acknowledgements. The authors are enormously grateful to the anonymous referee for his/her helpful comments.

\section{References}

[1] E. Artin, Quadratische Körper im Gebiete der höherer Kongruenzen I, Math. Z. 19 (1924), 153-206.

[2] L. Carlitz, A class of polynomials, Trans. Amer. Math. Soc. 43 (1938), 167-182.

[3] S. Galovich and M. Rosen, Units and class groups in cyclotomic function field, J. Number Theory 14 (1982), 156-184.

[4] D. Hayes, Explicit class field theory for rational function fields, Trans. Amer. Math. Soc. 189 (1974), 77-91.

[5] S. Lang, Algebra, 3rd rev. ed., Springer, New York, 1986.

[6] Y. Li and X. Zhang, Global unit squares and local unit squares, J. Number Theory 128 (2008), 2687-2694. 
[7] M. Rosen, Number Theory in Function Fields, Springer, New York, 2002.

[8] -, The Hilbert class field in function fields, Exp. Math. 5 (1987), 365-378.

[9] L. C. Washington, Introduction to Cyclotomic Fields, 2nd ed., Springer, New York, 1997.

Department of Mathematical Sciences

Tsinghua University

Beijing 100084, China

E-mail: hus04@mails.tsinghua.edu.cn

liyan_00@mails.tsinghua.edu.cn

Received on 10.3.2008

and in revised form on 12.2.2009 